\title{
Kinesin light chain-1 serine-460 phosphorylation is altered in Alzheimer's disease and regulates axonal transport and processing of the amyloid precursor protein
}

\author{
Gábor M. Mórotz ${ }^{1 \dagger}$, Elizabeth B. Glennon ${ }^{1 \dagger}$, Jenny Greig ${ }^{1}$, Dawn H. W. Lau', Nishita Bhembre ${ }^{1}$, Francesca Mattedi ${ }^{1}$,
} Nadine Muschalik ${ }^{2}$, Wendy Noble ${ }^{1}$, Alessio Vagnoni ${ }^{1 *}$ and Christopher C. J. Miller ${ }^{{ }^{*}}$ (D)

\begin{abstract}
Damage to axonal transport is an early pathogenic event in Alzheimer's disease. The amyloid precursor protein (APP) is a key axonal transport cargo since disruption to APP transport promotes amyloidogenic processing of APP. Moreover, altered APP processing itself disrupts axonal transport. The mechanisms that regulate axonal transport of APP are therefore directly relevant to Alzheimer's disease pathogenesis. APP is transported anterogradely through axons on kinesin-1 motors and one route for this transport involves calsyntenin-1, a type-1 membrane spanning protein that acts as a direct ligand for kinesin-1 light chains (KLCS). Thus, loss of calsyntenin-1 disrupts APP axonal transport and promotes amyloidogenic processing of APP. Phosphorylation of KLC1 on serine- 460 has been shown to reduce anterograde axonal transport of calsyntenin-1 by inhibiting the KLC1-calsyntenin-1 interaction. Here we demonstrate that in Alzheimer's disease frontal cortex, KLC1 levels are reduced and the relative levels of KLC1 serine-460 phosphorylation are increased; these changes occur relatively early in the disease process. We also show that a KLC1 serine-460 phosphomimetic mutant inhibits axonal transport of APP in both mammalian neurons in culture and in Drosophila neurons in vivo. Finally, we demonstrate that expression of the KLC1 serine-460 phosphomimetic mutant promotes amyloidogenic processing of APP. Together, these results suggest that increased KLC1 serine-460 phosphorylation contributes to Alzheimer's disease.
\end{abstract}

Keywords: Axonal transport, Alzheimer's disease, Kinesin-1, Kinesin light chain, Amyloid precursor protein, Calsyntenin-1, Drosophila melanogaster

\section{Introduction}

Intracellular transport of proteins, organelles and other cargoes is an essential requirement for vertebrate cell function. This is particularly so for neurons since most neuronal proteins are synthesised in cell bodies and then have to be transported to their final functional destinations in axons, dendrites and synapses $[6,10,15,19,31$,

\footnotetext{
* Correspondence: alessio.vagnoni@kcl.ac.uk; chris.miller@kcl.ac.uk

${ }^{\dagger}$ Gábor M. Mórotz and Elizabeth B. Glennon contributed equally to this work. 'Department of Basic and Clinical Neuroscience, Institute of Psychiatry,

Psychology and Neuroscience, King's College London, 125 Coldharbour Lane Camberwell, London SE5 9RX, UK

Full list of author information is available at the end of the article
}

36]. Notably, the distances over which cargoes have to be trafficked through axons which can be over a metre in length in humans, present unique challenges for neuronal transport systems. Indeed, damage to axonal transport is known to contribute to Alzheimer's disease, Parkinson's disease and motor neuron diseases [6, 10, $15,19,36]$.

Changes in metabolism of APP are believed to contribute to Alzheimer's disease; mutations in the APP gene cause some dominant familial forms of Alzheimer's disease and proteolytic processing of APP generates amyloid- $\beta$ peptide $(A \beta)$ which is deposited as a pathology in the brains of Alzheimer's disease patients [59].

(c) The Author(s). 2019 Open Access This article is distributed under the terms of the Creative Commons Attribution 4.0 International License (http://creativecommons.org/licenses/by/4.0/), which permits unrestricted use, distribution, and reproduction in any medium, provided you give appropriate credit to the original author(s) and the source, provide a link to the Creative Commons license, and indicate if changes were made. The Creative Commons Public Domain Dedication waiver (http://creativecommons.org/publicdomain/zero/1.0/) applies to the data made available in this article, unless otherwise stated. 
APP is a type- 1 membrane spanning protein and A $\beta$ production involves successive cleavage of APP by $\beta$-site APP cleaving enzyme-1 (BACE1) and $\gamma$-secretase which cleave at the $\mathrm{N}$ - and $\mathrm{C}$-termini respectively of the $\mathrm{A} \beta$ sequence. APP represents a key axonal transport cargo in Alzheimer's disease. This is because disruption to anterograde axonal transport of APP is an early feature of Alzheimer's disease, such disruption promotes amyloidogenic processing of APP and altered processing of APP itself perturbs axonal transport $[28,49,50,56]$. Damage to axonal transport of APP has therefore been proposed to induce a toxic cycle of events that eventually lead to neuronal cell death in Alzheimer's disease [50].

APP is transported anterogradely through axons on kinesin-1 motors [23, 50]. Most functional kinesin-1 comprises a heterotetramer of two kinesin-1 motor proteins (kinesin heavy chains) and two kinesin-1 light chains (KLCs). The heavy chains contain ATPase activity and generate motile force whereas the light chains (KLC1 and KLC2) are involved in attachment of cargoes [39]. A number of routes for attachment of APP to kinesin-1 motors have been described but a particularly important one involves the KLC ligand calsyntenin-1 (also known as alcadein- $\alpha$ ) [11, 13, 30, 49, 56]. Calsyntenin-1 is a type-1 membrane spanning protein that binds directly to KLCs via C-terminally located tryptophan-aspartate motifs that interact with the tetratricopeptide repeat domain of KLCs [4, 12, 26]. Axonal transport of APP involves its loading onto calsyntenin-1 containing vesicles in the Golgi [56]. Thus, loss of calsyntenin-1 inhibits anterograde axonal transport of APP and also promotes BACE1 cleavage of APP to increase $A \beta$ production $[30,49,56]$. Interestingly, calsyntenin-1 levels are reduced in Alzheimer's disease brains and this suggests that calsyntenin- 1 loss contributes to damaged APP transport in Alzheimer's disease [56].

The mechanisms that underpin loading and release of APP containing vesicles to and from kinesin- 1 motors are not properly understood but clearly represent key regulatory routes for controlling APP axonal transport. Notably, phosphorylation of serine-460 in KLC1 has been shown to inhibit its interaction with calsyntenin-1 leading to a reduction in calsyntenin-1 axonal transport [55]. KLC1 serine-460 is highly evolutionarily conserved $[18,55]$. Moreover, these effects appear specific for calsyntenin-1 since KLC1 serine- 460 phosphorylation does not influence binding and transport of a number of other KLC1 ligands [55]. Thus, via its effect on calsyntenin-1 binding, phosphorylation of KLC1 serine460 may also regulate axonal transport of APP. Here, we address the role of KLC1 serine- 460 phosphorylation in APP axonal transport and processing, and in Alzheimer's disease.

\section{Materials and methods}

\section{Plasmids}

pCI-neo control empty vector was from Promega. Human APP (isoform 695) in pCI-neo, enhanced green fluorescent protein (EGFP)-tagged human APP (isoform 695) and FLAG-tagged wild-type KLC1 (isoform A; KLC1wt), and KLC1 in which serine-460 was mutated to aspartic acid (KLC1S460D) or alanine (KLC1S460A) have all been described previously [9, 43, 55]. Human APP isoform 695 fused in frame at its C-terminus to the yeast transcription factor GAL4 (APP-GAL4) was as described [22]. pG5-Luc in which firefly luciferase is driven by a GAL4 dependent promoter and pRL-CMV which expresses control Renilla luciferase were from Promega.

\section{Antibodies}

The following primary antibodies were used in this study:

Mouse anti-total extracellular-signal-regulated kinase 1/2 (ERK1/2) (L34F12 Cell Signaling 1/2000), rabbit anti-active ERK1/2 (phosphorylated on threonine-202 and tyrosine-204) (Cell Signaling 1/2000), mouse antiFLAG (M2 Sigma 1/2000), rabbit anti-total KLC1 (sc25735/H-75 Santa Cruz 1/2000), mouse anti-neuron specific enolase (NSE Dako 1/50,000), mouse antitubulin (DM1A Sigma 1/20,000). Rabbit antibody to KLC1 phosphorylated on serine- 460 was generated by immunisation with keyhole limpet hemocyanin coupled to peptide CKVDS ${ }^{\text {phos }}$ PTVTTTLKNL in which serine$460\left(\mathrm{~S}^{\text {phos }}\right)$ was phosphorylated (Proteintech). The antibody was affinity purified against the peptide prior to use. Rabbit anti-total KLC1 (sc-25735/H-75) has been utilised in numerous previous studies e.g. [37, 41].

\section{Cell culture and transfection}

Human embryonic kidney-293 (HEK293) cells were grown in Dulbecco's modified Eagle's medium with 4.5 $\mathrm{g} / \mathrm{l}$ glucose (GE Healthcare) supplemented with $10 \%$ (v/ v) fetal bovine serum and $2 \mathrm{mML}$-glutamine. Cells were transfected using TurboFect (Thermo Scientific) or with polyethylenimine MAX (Polysciences) according to the manufacturer's instructions. Cells were analysed $24 \mathrm{~h}$ post-transfection. Okadaic acid was from Santa Cruz and applied at $50 \mathrm{nM}$ for $4 \mathrm{~h}$.

Rat cortical neurons were obtained from embryonic day 17 embryos, plated on poly-L-lysine coated $18 \mathrm{~mm}$ diameter glass coverslips (Marienfield GmbH \& Co.KG) and cultured in Neurobasal medium containing B27 supplement (Invitrogen), $2 \mathrm{mM}$ L-glutamine, $100 \mathrm{IU} / \mathrm{ml}$ penicillin and $100 \mu \mathrm{g} / \mathrm{ml}$ streptomycin. Neurons were transfected using Lipofectamine 2000 (Invitrogen) $(2 \mu \mathrm{l} /$ $\mu \mathrm{g}$ DNA in Opti-MEM) according to the manufacturer's instructions and analysed $24 \mathrm{~h}$ post transfection on DIV6 or 7. 


\section{Preparation of human brain samples for SDS-PAGE and immunoblotting}

Post-mortem human frontal cortex samples from control and pathologically confirmed cases of Alzheimer's disease were obtained from the Medical Research Council Neurodegenerative Diseases Brain Bank, King's College London. All tissue collection and processing were carried out under the regulations and licensing of the Human Tissue Authority, and in accordance with the Human Tissue Act, 2004. Post-mortem studies from some control, clinically non-demented individuals revealed early Braak stage pathologies. Frozen human brain tissues were prepared as $20 \%$ homogenates in icecold radioimmunoprecipitation assay (RIPA) buffer (50 mM Tris- $\mathrm{HCl}, \mathrm{pH} 7.4 ; 150 \mathrm{mM} \mathrm{NaCl} ; 1 \mathrm{mM}$ EDTA; $1 \%$ (v/v) Triton X-100; $0.5 \%(\mathrm{w} / \mathrm{v})$ sodium deoxycholate; $0.1 \%(\mathrm{w} / \mathrm{v}) \mathrm{SDS}$ ) with protease and phosphatase inhibitor cocktails (Complete and PhosStop, Roche) using a BioGen PRO200 rotor-stator homogeniser (Pro Scientific) for $20 \mathrm{~s}$. Following homogenisation, each sample was sonicated three times for $3 \mathrm{~s}$ before being centrifuged at $13,000 \mathrm{x} \mathrm{g}$ for $20 \mathrm{~min}$ at $4{ }^{\circ} \mathrm{C}$. Supernatants were collected and protein concentrations determined using a bicinchoninic acid protein concentration assay kit (Pierce) according to the manufacturer's instructions. Protein concentrations were adjusted to the same concentration in each sample by adding RIPA and SDSPAGE sample buffers. Samples were stored at $-80^{\circ} \mathrm{C}$.

\section{SDS-PAGE and immunoblotting}

Cells were harvested for SDS-PAGE and immunoblotting by scraping into SDS-PAGE sample buffer containing $2 \%(\mathrm{w} / \mathrm{v})$ SDS, $100 \mathrm{mM}$ dithiothreitol, 10\% (w/v) glycerol, $0.1 \%(\mathrm{w} / \mathrm{v})$ bromophenol blue plus protease in $50 \mathrm{mM}$ Tris- $\mathrm{HCl} \mathrm{pH} 6.8$ and heating to $96^{\circ} \mathrm{C}$ for $5 \mathrm{~min}$. Human post-mortem brain samples were heated to $96{ }^{\circ} \mathrm{C}$ for $10 \mathrm{~min}$ prior to SDS-PAGE. Samples were separated on 8 or $12 \%$ gels using Mini-PROTEAN 3 gel electrophoresis systems (Bio-Rad) with a discontinuous buffer system. Separated proteins were transferred to BioTrace NT nitrocellulose membrane $(0.2 \mu \mathrm{m}$ pore size; Pall Corporation) using a Mini Trans-Blot electrophoretic transfer cell (Bio-Rad) for $16 \mathrm{~h}$. Membranes were blocked with Tris-HCl buffered saline (TBS) containing either $5 \%(\mathrm{w} / \mathrm{v})$ milk powder or $5 \%(\mathrm{w} / \mathrm{v})$ bovine serum albumin, or with Odyssey TBS blocking buffer (Li-Cor Biosciences) for $1 \mathrm{~h}$. Membranes were probed with primary antibodies in blocking buffers supplemented with $0.1 \%(\mathrm{w} / \mathrm{v}$ ) Tween-20 (TBS/Tween-20), washed in TBS/ Tween-20 and incubated with horseradish peroxidase (HRP)-conjugated secondary antibodies in wash buffer, and developed using an enhanced chemiluminescence development reagent (GE Healthcare) and detected using a BioRad ChemiDoc MP Imaging system.
Alternatively, blots were incubated with IRDyeconjugated secondary antibodies in wash buffer and proteins visualised using an Odyssey CLx near infrared imaging system (Li-Cor Biosciences). KLC1 signals obtained from human brain samples were normalised to NSE signals from the same membrane. Both the BioRad ChemiDoc MP and Odyssey CLx near infrared imaging systems provide signals within the linear range and only such values were used for quantification.

\section{Drosophila melanogaster studies}

All Drosophila stocks were cultured on Iberian food as described [53]. The following Drosophila strains were obtained from the Bloomington Drosophila Stock Center (Indiana University, IN): Appl-Gal4 (BL\#32040); UASAPP::YFP (BL\#32039); nos-phiC31int.NLS (attP40, BL\#25709); $w^{1118}$ (BL\#5905). TM3/TM6b and nos-Cas9 (BL\#54591) stocks were gifts from Simon Bullock (MRC-LMB Cambridge). nos-Cas9 and nos-phiC31int.NLS Drosophila were sequenced across the target region of the $K l c$ gene to ensure no polymorphisms were present compared to the reference genome sequence that might interfere with the production of mutant $K l c$ Drosophila.

KLC1 serine-460 and surrounding sequences are highly evolutionarily conserved and in Drosophila the homologous residue is KLC serine-433 [18, 55]. Drosophila KLC serine-433 was altered to aspartate using type II clustered regularly interspaced short palindromic repeat (CRISPR)/CRISPR-associated (Cas) mutagenesis. The guide RNA (gRNA) protospacer sequence directing Cas9-mediated cleavage was introduced by annealing the following oligonucleotides 5' - GTCGTGGCATAAGG CCGCTAAAG-3' (top strand) and 5'-AAACCTTTAG CGGCCTTATGCCA-3' (bottom strand) into the BbsI site of plasmid pCDF3 [44]. Potential off-target hits were evaluated using CRISPR target finder (http://tools.flycrispr.molbio.wisc.edu/targetFinder/) and E-CRISP (www.e-crisp.org/E-CRISP/). The gRNA efficiency score was calculated with the CRISPR Efficiency Predictor (http://www.flyrnai.org/evaluateCrispr/). The $g R N A-K l c$ construct was integrated into the attP40 (25C6) landing site by phiC31 integrase-mediated transgenesis following embryo injection.

The single stranded DNA oligonucleotide donor (ssODN) for homology-directed repair was designed to anneal to an asymmetric region $-91 /+36$ bp (i.e. proximal/distal) from the protospacer adjacent motif (PAM) site and complementary to the "target" strand (i.e. the strand targeted by the gRNA) [46]. The ssODN sequence was $5^{\prime}$-CATATGGCGAGTACGGCGGTTG GCATAAGGCCGCTAAAGTAGATGACCCCACGGT CACAACCACTCTAAAAAATCTGGGAGCACTTTAC CGACGTCAAGGCATGTTTGAAGCGGCCGAAACC 
CTGGA-3' (4 nM Ultramer ${ }^{\circ}$ DNA, Integrated DNA Technologies). The PAM site was mutated to prevent further Cas9 cleavage after the introduction of the desired mutation without a change to the amino acid sequence of the product. The ssODN was delivered in noscas9/+; U6-3-gRNA-Klc/+ embryos 0.5-1 h after egg laying as a $500 \mathrm{ng} / \mu \mathrm{l}$ solution in $\mathrm{H}_{2} \mathrm{O}$ as previously described [44].

To identify KLCS433D mutant Drosophila, 4 G0 flies derived from the ssODN injected embryos were crossed to the TM3/TM6b balancer stock and the progeny of these flies screened by direct sequencing of the Klc gene. Briefly, a $582 \mathrm{bp}$ region of the Klc gene encompassing the mutant site was amplified by PCR and sequenced as described [44]. The primer sequences were 5'-AAGCAACTTAACAA TCTCGCCCTGCTC-3' (Forward) and 5'-CGCATT CTTCTCCTCAGAGAAATCCAAATCC-3' (Reverse). All founder animals and 12 of 23 offspring (52\%) transmitted the mutation. G2 animals bearing the desired mutation were then backcrossed for 10 generations to an isogenic $w^{1118}$ strain to minimise the possibility of off-target effects due to non-specific binding of the gRNA. During backcrossing, direct DNA sequencing of a PCR generated region of Klc was again used to identify mutant Drosophila.

For imaging of APP-YFP in the homozygous KLCS433D background (termed KlcS433D), Appl-Gal4; KlcS433D/TM3 virgin females were crossed with $U A S$ APP::YFP/CyO; KlcS433D/TM3 males. The control genotype involving wild-type $K l c$ was generated by crossing Appl-Gal4 virgin females to UAS-APP::YFP males.

Quantification of APP transport by time-lapse microscopy Axonal transport of APP-EGFP in living rat cortical neurons was monitored essentially as described previously for analyses of APP-EGFP and other fluorescent proteintagged cargoes [1, 40, 41, 55-57]. APP-EGFP was imaged using either a Zeiss Axiovert S100 microscope driven by MetaMorph (Molecular Dynamics) and a 40x Plan-Neofluar 1.3NA objective, and a Photometrics Cascade-II 512B36 electron-multiplying charge-coupled device camera or alternatively, a Nikon Eclipse Ti-E microscope driven by NIS-Elements AR software and equipped with Intenslight C-HGFI light source, CFI Apo Lambda S 60x/1.40NA objective and an Andor Neo scientific complementary metal-oxide-semiconductor camera (Andor Technology) [41, 56, 57]. Filter sets were from Chroma Technology. APP-EGFP was imaged 24$36 \mathrm{~h}$ post-transfection in Ibidi $\mu$-dishes or by mounting coverslips in a Ludin imaging chamber (Life Imaging Services) filled with external solution $(145 \mathrm{mM} \mathrm{NaCl}, 2$ $\mathrm{mM} \mathrm{KCl}, 5 \mathrm{mM} \mathrm{NaHCO}, 1 \mathrm{mM} \mathrm{MgCl}, 2.5 \mathrm{mM} \mathrm{CaCl}$, $10 \mathrm{mM}$ glucose in $10 \mathrm{mM}$ HEPES pH 7.0). Temperature was maintained at $37^{\circ} \mathrm{C}$ during imaging using either a Box Microscope temperature control system (Life
Imaging Systems) for the Zeiss microscope or a microscope incubation chamber (Solent Scientific) for the Nikon microscope. Movements were recorded at $1 \mathrm{~s}$ time-lapse intervals and $100 \mathrm{~ms}$ exposure times. Kymographs were created using the Straighten and Kymograph plugins of ImageJ (developed by Wayne Rasband, National Institute of Health, Bethesda USA). Overall velocities for each run were calculated using the KymoAnalyser ImageJ macro package [42]. In line with previous studies, we chose cells expressing low levels of transfected APP-EGFP (as judged by the fluorescent protein signal) for analyses so as to avoid any possible artefacts produced by high levels of expression [1, 40, 41, 55-57].

APP-YFP movement was monitored in vivo in adult Drosophila sensory wing neurons essentially as described for other fluorescent protein-tagged cargoes [53, 54, 58]. Images were captured using a Nikon spinning disk system with a CSU-X1 scanning head (Yokogawa) and a Nikon Eclipse Ti-E inverted microscope equipped with a $60 x \mathrm{xFI}$ Apo/1.4NA objective and a $\mathrm{Du} 897$ iXon Ultra electron-multiplying chargecoupled device camera (Andor). APP-YFP movement was monitored for $2 \mathrm{~min}$ with an acquisition rate of 1 frame/s. A temperature of $25^{\circ} \mathrm{C}$ was maintained throughout imaging with a temperature unit and microscope enclosure (Okolab). Kymographs of APPYFP were generated in Fiji/ImageJ with the KymoClear toolset from a $70 \mu \mathrm{m}$ axonal region [32]. APPYFP run numbers (defined as unidirectional movement in either anterograde or retrograde direction) and velocities were automatically analysed from the kymographs using the KymoDirect tool [32, 58]. Accuracy of the tracking was confirmed by manual inspection. Equal numbers of male and females were analysed and the data were derived from at least two independent Drosophila crosses.

\section{Luciferase reporter assays to monitor amyloidogenic APP processing}

Amyloidogenic processing of APP was monitored in HEK293 cells essentially as described using an APPGAL4 reporter assay that drives GAL4 upstream activator sequence (GAL4-UAS)-dependent expression of firefly luciferase [22]. Briefly, APP fused in frame at its Cterminus to GAL4 was co-transfected into HEK293 cells with a GAL4-UAS-firely luciferase reporter gene (pFRLuc), Renilla transfection efficiency control plasmid (pRL-CMV) and either KLC1wt or KLC1S460D. BACE1, $\alpha$-secretase and $\gamma$-secretase cleavage of APP releases the APP intracellular domain fused to GAL4 which translocates to the nucleus to regulate expression of firefly luciferase. Transfection efficiency normalised firefly luciferase signals thus provide a readout for amyloidogenic processing of APP. Cells were transfected in 24 
well plates at $70-80 \%$ confluence with $0.25 \mu \mathrm{g}$ each of APP-GAL4, pFR-Luc and either KLC1wt or KLC1S460D, along with $0.025 \mu \mathrm{g}$ of pRL-CMV Renilla control plasmid. Cells were transfected as described above (see Cell culture and transfection). Luciferase signals were developed $24 \mathrm{~h}$ later using a Dual-Glo Luciferase assay kit (Promega) according to the manufacturer's instructions and quantified using a Promega GloMax Navigator luminometer. Full details of the assay including design and construction of plasmids have been described previously [22].

\section{$A \beta$ assays}

$A \beta$ (1-38), $A \beta(1-40)$ and $A \beta(1-42)$ levels were measured in conditioned media from HEK293 cells cotransfected with APP+KLC1wt or APP+KLC1S460D using a V-PLEX Plus A $\beta$ peptide panel 1 (6E10) kit (Meso Scale Discovery) according to the manufacturer's instructions. Cells were co-transfected in 24 well plates at $70-80 \%$ confluence with $0.25 \mu \mathrm{g}$ of each plasmid as described above (see Cell culture and transfection). Conditioned media was harvested $24 \mathrm{~h}$ post-transfection and centrifuged at $1200 \mathrm{x} \mathrm{g}$ for $5 \mathrm{~min}$ at $4{ }^{\circ} \mathrm{C}$. Supernatants were transferred into an ELISA plate and incubated for $1 \mathrm{~h}$ to capture $A \beta$ species. Plates were then washed three times with PBS supplemented with $0.05 \%(\mathrm{v} / \mathrm{v})$ Tween20 and incubated with detection antibody for $2 \mathrm{~h}$. Following three washes, read buffer was added to the samples and plates were analysed using a Meso Sector S 600 plate reader (Meso Scale Discovery).

\section{Statistical analyses}

Statistical analysis was performed using Excel (Microsoft Corporation) and Prism software (version 7.02; GraphPad Software Inc.). Statistical significance was determined by t-tests or analyses of variance (ANOVA) followed by post-hoc test as described in the figure legends.

\section{Results}

KLC1 levels are reduced and the relative levels of KLC1 serine-460 phosphorylation are increased in post-mortem Alzheimer's disease frontal cortex

To facilitate studies of KLC1 serine-460 phosphorylation in human Alzheimer's post-mortem tissues, we generated a rabbit polyclonal antibody that recognises phosphorylation of KLC1 on this residue by immunisation with a KLC1 peptide in which serine-460 was phosphorylated. To demonstrate the specificity of this antibody, we transfected HEK293 cells with control vector, FLAGKLC1wt or FLAG-KLC1S460A in which serine-460 was mutated to alanine to preclude phosphorylation. We also treated FLAG-KLC1wt transfected cells with the serine/ threonine phosphatase inhibitor okadaic acid which activates ERK so as to increase KLC1 serine- 460 phosphorylation; KLC1 serine-460 is targeted by ERK [55]. Probing of these samples on immunoblots with the KLC1 serine-460 phospho-specific antibody revealed that okadaic acid treatment increased FLAG-KLC1 signals but that signals were abolished in FLAGKLC1S460A transfected cells (Fig. 1). These data are consistent with the antibody specifically recognising KLC1 phosphorylated on serine-460.

We next utilised this antibody to enquire whether KLC1 serine- 460 phosphorylation might be altered in Alzheimer's disease. To do so, we monitored both total and serine-460 phosphorylated KLC1 levels in postmortem control and Alzheimer's disease frontal cortex tissues by immunoblotting. Details of these human samples are shown in Table 1; there were no significant differences in age or post-mortem delay between the Alzheimer's disease and control cases. We studied total KLC1 and serine-460 phosphorylated KLC1 levels in frontal cortex in control, Braak stage III-IV (mid dementia) and Braak stage VI (severe dementia) cases. We normalised total and phosphorylated KLC1 levels to the levels of NSE as described by others [27, 41, 52]. Compared to controls, total KLC1 levels were significantly reduced in frontal cortex in both Braak stage III-IV and Braak stage VI cases (Fig. 2). Moreover, following normalisation to total KLC1 levels, the levels of KLC1

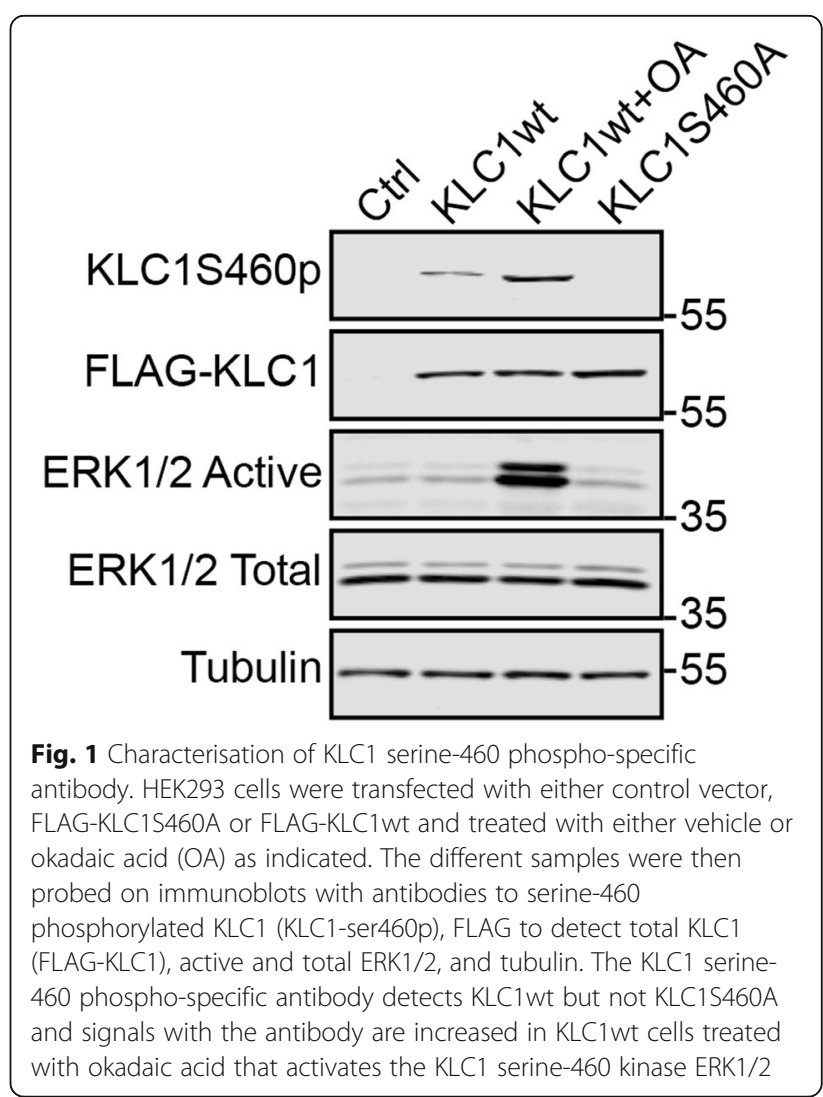


Table 1 Data for human post-mortem samples showing age at death, sex, post-mortem delay and pathological diagnosis

\begin{tabular}{|c|c|c|c|c|c|}
\hline Case Group & Autopsy Code & Sex & Age & Post-Mortem Delay (h) & Braak stage \\
\hline Control & $\mathrm{A} 002 / 13$ & M & 90 & 45 & - \\
\hline Control & $\mathrm{A} 007 / 15$ & $\mathrm{~F}$ & 74 & 64 & $\|$ \\
\hline Control & $\mathrm{A} 033 / 11$ & M & 82 & 47 & 1 \\
\hline Control & $\mathrm{A} 046 / 12$ & F & 92 & 9 & $\|$ \\
\hline Control & A063/10 & $F$ & 90 & 50 & $\|$ \\
\hline Control & A105/14 & F & 77 & 21 & - \\
\hline Control & A158/14 & $\mathrm{F}$ & 73 & 27 & । \\
\hline Control & A209/13 & M & 80 & 55 & $\|-\mid\|$ \\
\hline Control & A213/12 & M & 78 & 24 & III \\
\hline Control & A308/09 & M & 66 & 52 & - \\
\hline Control & A319/14 & $\mathrm{F}$ & 90 & 44 & $\|$ \\
\hline Control & A346/10 & $F$ & 84 & 34 & $|-| \mid$ \\
\hline Control & A359/08 & F & 80 & 3 & I \\
\hline Control & A407/13 & F & 80 & 22 & $\|$ \\
\hline AD Braak III-IV & A037/04 & F & 96 & 39 & IV \\
\hline AD Braak III-IV & A041/04 & F & 97 & 67.5 & III-IV \\
\hline AD Braak III-IV & A065/16 & M & 91 & 48 & IV \\
\hline AD Braak III-IV & A067/09 & $F$ & 92 & 19.5 & III \\
\hline AD Braak III-IV & A078/13 & M & 86 & 52.5 & IV \\
\hline AD Braak III-IV & A097/13 & M & 82 & 28 & IV \\
\hline AD Braak III-IV & A101/08 & $\mathrm{F}$ & 92 & 29.5 & IV \\
\hline AD Braak III-IV & A189/07 & F & 83 & 41.5 & IV \\
\hline AD Braak III-IV & A223/12 & F & 83 & 22 & IV \\
\hline AD Braak III-IV & A232/16 & F & 95 & 47 & IV \\
\hline AD Braak III-IV & A282/11 & M & 93 & 13.5 & IV \\
\hline AD Braak III-IV & A374/14 & M & 88 & 79 & III-IV \\
\hline AD Braak III-IV & A378/14 & M & 98 & 53 & IV \\
\hline AD Braak III-IV & A381/16 & M & 84 & 86 & IV \\
\hline AD Braak VI & $\mathrm{A} 008 / 12$ & M & 66 & 41 & $\mathrm{Vl}$ \\
\hline AD Braak VI & A064/16 & F & 93 & 49 & $\mathrm{Vl}$ \\
\hline AD Braak VI & A087/16 & $F$ & 89 & 38.5 & $\mathrm{Vl}$ \\
\hline AD Braak VI & $\mathrm{A} 100 / 15$ & $\mathrm{~F}$ & 73 & 30 & $\mathrm{Vl}$ \\
\hline AD Braak VI & A105/13 & F & 81 & 17.5 & $\mathrm{Vl}$ \\
\hline AD Braak VI & A166/12 & F & 86 & 25 & $\mathrm{Vl}$ \\
\hline AD Braak VI & A171/14 & M & 84 & 67 & $\mathrm{Vl}$ \\
\hline AD Braak VI & A226/16 & $\mathrm{F}$ & 69 & 73 & $\mathrm{Vl}$ \\
\hline AD Braak VI & A258/16 & M & 67 & 39.5 & $\mathrm{Vl}$ \\
\hline AD Braak VI & A289/13 & M & 83 & 22 & $\mathrm{Vl}$ \\
\hline AD Braak VI & A331/15 & M & 86 & 38 & $\mathrm{Vl}$ \\
\hline AD Braak VI & A342/14 & $F$ & 84 & 27 & $\mathrm{Vl}$ \\
\hline AD Braak VI & A355/14 & F & 79 & 31 & $\mathrm{Vl}$ \\
\hline AD Braak VI & A377/14 & F & 85 & 79 & $\mathrm{Vl}$ \\
\hline AD Braak VI & A380/13 & F & 81 & 20 & $\mathrm{VI}$ \\
\hline
\end{tabular}

$A D$, Alzheimer's disease; $F$, female; $M$, male 


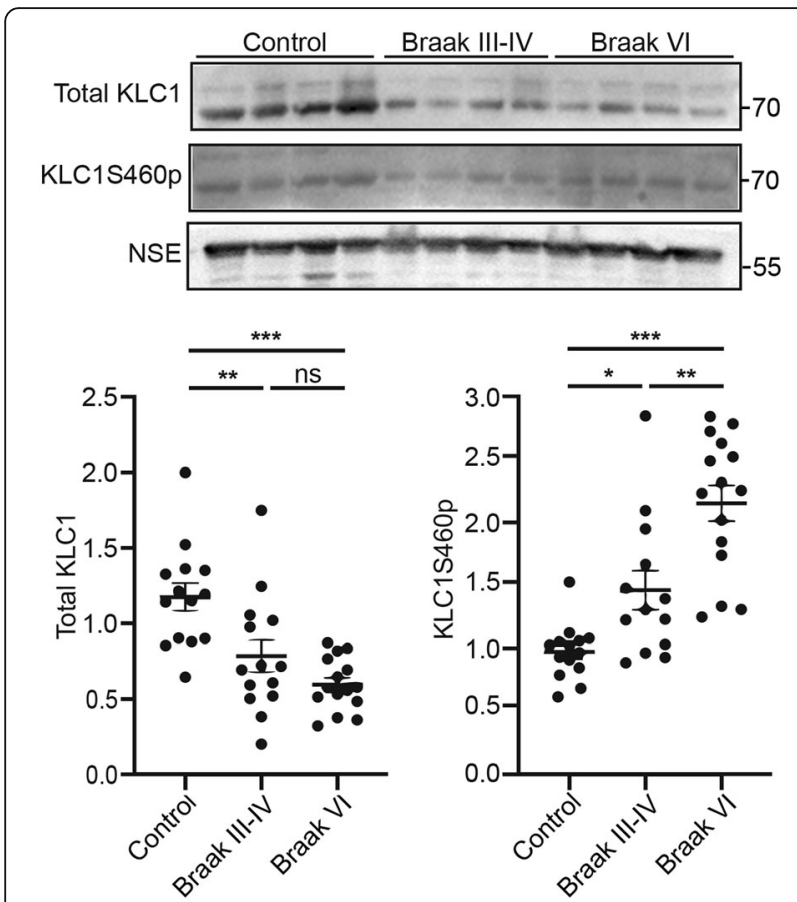

Fig. $2 \mathrm{KLC} 1$ levels are reduced and the relative levels of KLC1 serine460 phosphorylation are increased in Alzheimer's disease frontal cortex. Representative immunoblots showing total KLC1, KLC1 serine-460 phosphorylation (KLC1S460p) and NSE levels in postmortem human control (Ctrl) and Alzheimer's disease frontal cortex. Braak stages are indicated. Graphs show quantification of total KLC1 and KLC1 phosphorylated on serine-460 in the different samples. $\mathrm{KLC1}$ and KLC1S460p signals were normalised to NSE levels. These normalised KLC1 levels were then used to quantify changes to total KLC1 and KLC1S460p (expressed as the ratio of KLC1S460p/total KLC1). Data were analysed by Welch's ANOVA and Games-Howell post hoc test. $N=13-15$, error bars are s.e.m., ${ }^{*} p<0.05{ }^{*} p<0.01$, ${ }^{* * *} p<0.001$, ns not significant

phosphorylated on serine-460 were increased in both Braak stage III-IV and Braak stage VI cases (Fig. 2). These data suggest that both loss of KLC1 and increased KLC1 serine-460 phosphorylation may contribute to damaged APP axonal transport in Alzheimer's disease.

\section{Mutation of KLC1 serine- 460 to mimic permanent phosphorylation disrupts axonal transport of APP in cultured rat cortical neurons}

Reduction of KLC1 levels has been shown to impair APP axonal transport in rodent and Drosophila neurons and to exacerbate Alzheimer's disease phenotypes [16, $45,50]$. To investigate how phosphorylation of KLC1 serine-460 might contribute to Alzheimer's disease, we quantified axonal transport of APP-EGFP using timelapse microscopy in living rat cortical neurons that were co-transfected with either FLAG-KLC1wt or FLAGKLC1S460D in which serine-460 was mutated to aspartic acid to mimic permanent phosphorylation. There are many examples whereby replacing serine residues with a negatively charged residue such as aspartic acid accurately mimics the effect of phosphorylation of the site (e.g. [1, 2, 14, 34]. Indeed, mutation of KLC1 serine-460 to aspartate has already been shown to accurately mimic phosphorylation of this site so as to reduce KLC1 binding to calsyntenin-1 [55]. Kymographs were generated from the time-lapse movies, and these were used to calculate the percentages of total, anterograde and retrograde moving APP-EGFP cargoes as previously described [41, 50, 56, 57].

In APP-EGFP+FLAG-KLC1wt neurons, APP-EGFP movement was predominantly anterograde with mean speeds of $1.57+/-0.95 \mu \mathrm{m} / \mathrm{s}$ in the anterograde direction and $0.81+/-0.58 \mu \mathrm{m} / \mathrm{s}$ in the retrograde direction (mean+/-SD). These velocities and the bias towards anterograde movement are similar to those described previously for APP movement in rodent cortical neurons $[51,56]$. However, compared to FLAG-KLC1wt cotransfected neurons, total (anterograde plus retrograde) APP-EGFP movement was significantly reduced in FLAG-KLC1S460D co-transfected neurons (Fig. 3a). Analyses of individual anterograde and retrograde transport revealed that although expression of FLAGKLC1S460D appeared to reduce APP-EGFP transport in both directions, these reductions did not reach statistical significance (Fig. 3a). We also compared the anterograde and retrograde velocities of APP-EGFP movement in FLAG-KLC1wt and FLAG-KLC1S460D co-transfected neurons but detected no significant differences (Fig. 3b). Thus, compared to FLAG-KLC1wt co-transfected neurons, co-transfection of FLAG-KLC1S460D to mimic permanent phosphorylation inhibits total APP-EGFP axonal transport but does not alter the velocities of movement.

Mutation of endogenous Drosophila KLC serine-433, the homologue of mammalian serine- 460 , to mimic permanent phosphorylation disrupts axonal transport of APP in vivo

Drosophila KLC displays a high degree of sequence and structural homology with mammalian KLC1 [18] and KLC1 serine-460 and flanking sequences are highly conserved between mammals and Drosophila (Fig. 4a). To complement the above studies in rat cortical neurons, we therefore analysed how mutation of the Drosophila homologue of mammalian KLC1 serine-460 (KLC serine-433) to mimic permanent phosphorylation (KLCS433D) affects APP axonal transport in vivo. Drosophila KLC serine-433 was mutated to aspartic acid by CRISPR/Cas9-mediated homology-directed repair (Fig. 4b). We then compared APP-YFP transport in transgenic Drosophila in which endogenous KLC was either wild-type or homozygous for KLCS433D. APP-YFP axonal transport was monitored in vivo in adult living 

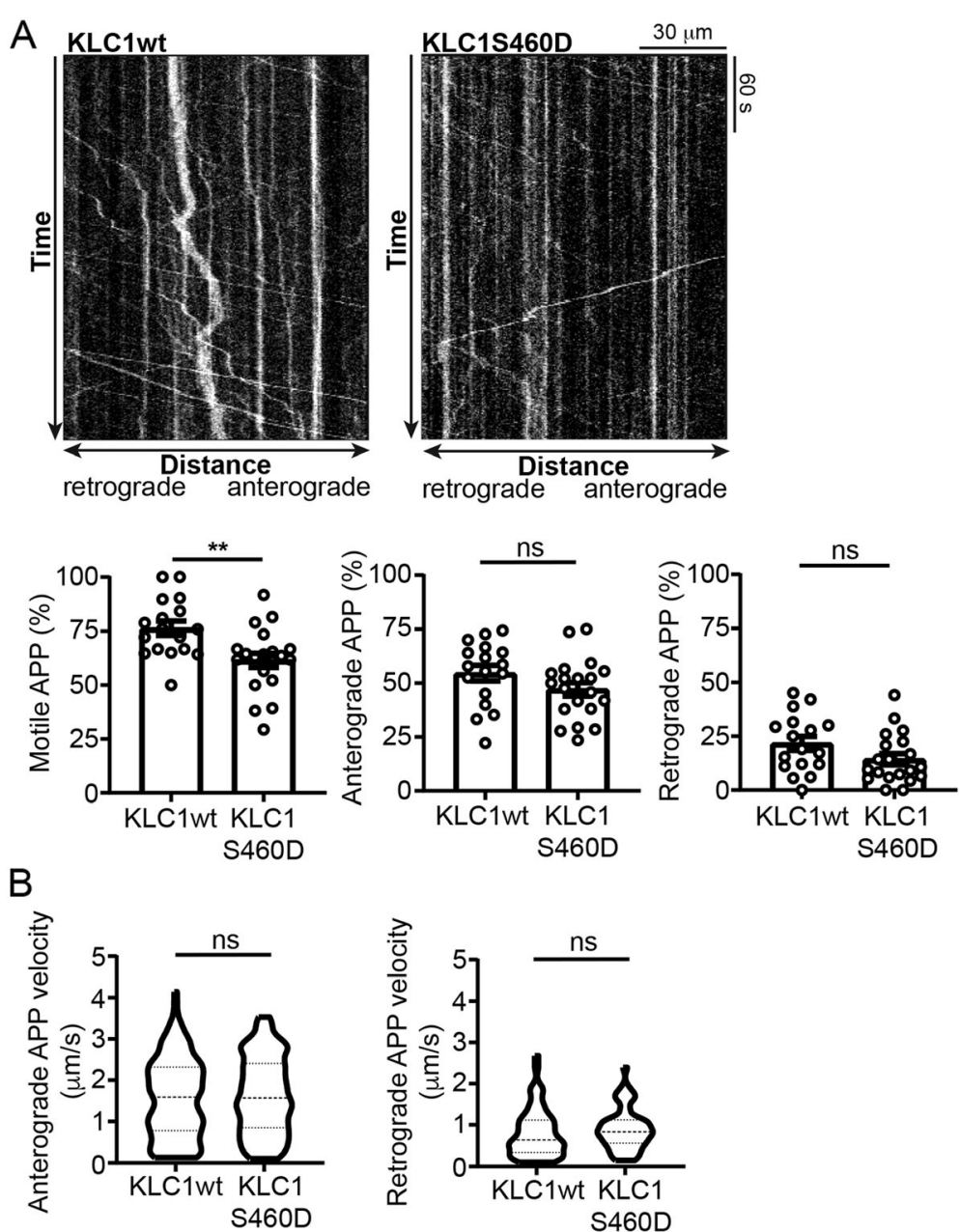

Fig. 3 Mutation of KLC1 serine-460 to mimic permanent phosphorylation, inhibits axonal transport of APP in cultured rat cortical neurons. (a) Representative kymographs showing axonal transport of APP-EGFP in APP-EGFP+KLC1wt and APP-EGFP+KLC1S460D co-transfected neurons; scale bar and times are indicated. Bar charts show \% for total, anterograde and retrograde APP-EGFP movement. N=17 EGFP+KLC1wt and 20 APPEGFP+KLC1S460D co-transfected neurons. Statistical significance was determined by Student's t-test. Error bars are s.e.m.; ${ }^{* *} p<0.01$; ns not significant. (b) Violin plots show velocities of APP-EGFP movement in anterograde and retrograde directions in the different transfected cells. Median and interquartile ranges are indicated by hashed lines. N = 163 anterogradely and 62 retrogradely moving APP-EGFP in KLC1 wt and 179 anterogradely and 54 retrogradely moving APP-EGFP in KLC1S460D co-transfected cells. Statistical significance was determined by Mann-Whitney $U$ test; ns not significant

sensory wing neurons essentially as described for other cargoes [53, 54].

In wild-type Drosophila, APP-YFP movement was bidirectional with mean speeds of $0.64+/-0.01 \mu \mathrm{m} / \mathrm{s}$ in the anterograde and $0.61+/-0.01 \mu \mathrm{m} / \mathrm{s}$ in the retrograde direction (mean+/-s.e.m.) (Fig. 4c). These transport characteristics are in line with previous findings of APP-YFP movement in Drosophila larvae segmental nerves [20, 50]. Anterograde and retrograde APP-YFP velocities were unaffected by the KlcS433D mutation (Fig. 4d). However, compared to wild-type Drosophila, both total and anterograde APP-YFP transport were significantly reduced in the $K l c S 433 D$ mutants (Fig. 4d). Retrograde APP-YFP transport also displayed some reduction in
KlcS433D mutant Drosophila but this did not reach significance. Thus, mutation of mammalian KLC1 serine460 and its Drosophila homologue KLC serine-433 to mimic permanent phosphorylation inhibit axonal transport of APP in both mammalian cultured neurons and in vivo in Drosophila.

\section{Expression of mutant KLC1 serine-460 to mimic permanent phosphorylation promotes amyloidogenic processing of APP}

Disruption to kinesin-1 mediated APP transport has been shown to promote amyloidogenic processing of APP $[28,49,50,56]$. We therefore monitored the effect of KLC1wt and KLC1S460D expression on APP 


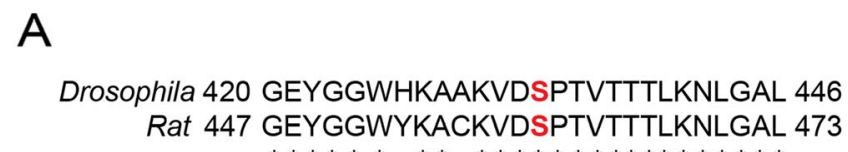

$* * * * * * * * * * * * * * * * * * * * * * * * *$

B

Klc: $\quad$ '-GTTGGCATAAGGCCGCTAAAGTGGGATṬ G̦CCC-3'

ssODN: 18nt-GTTGGCATAAGGCCGCTAAAGTÄGATGÄĊCCC-77nt

C

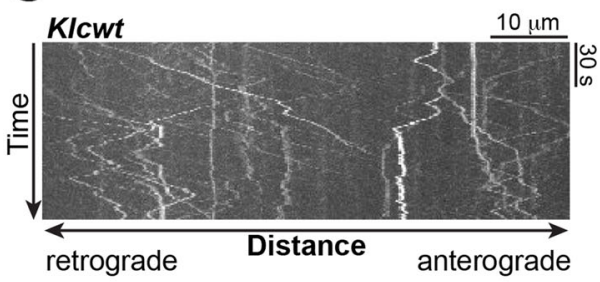

KICS433D
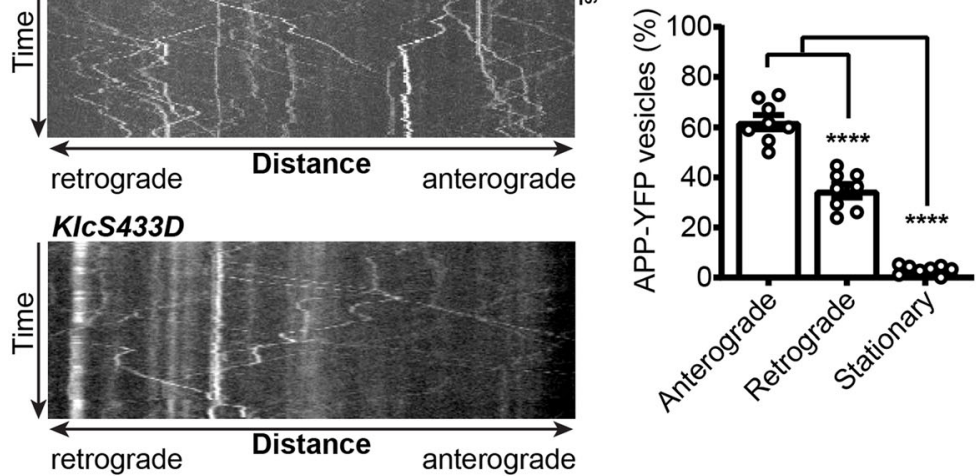

D
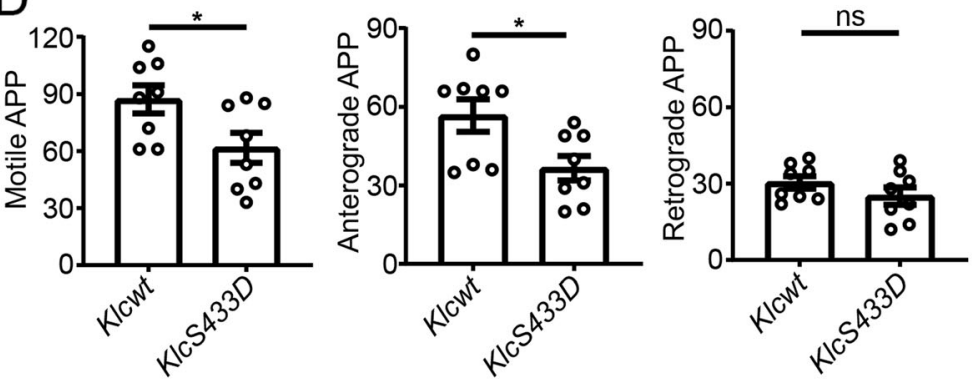

E
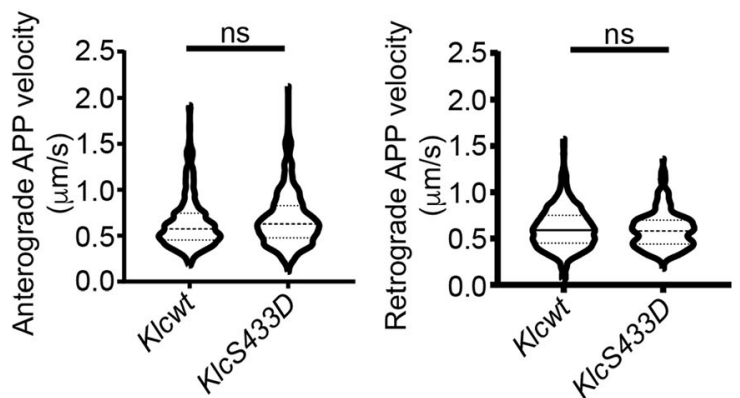

Fig. 4 (See legend on next page.)

processing using an APP-GAL4 dependent firefly luciferase reporter assay in which luciferase signals provide a readout for APP processing. $\gamma$-secretase processing of APP releases the APP intracellular domain fused to GAL4 which translocates to the nucleus to drive expression of a GAL4-UAS luciferase reporter [22]. Cells were co-transfected with APP-GAL4, firefly luciferase reporter and Renilla transfection efficiency control plasmids plus either KLC1wt or KLC1S460D. Compared to the KLC1wt co-transfected cells, transfection of KLC1S460D induced a significant increase in luciferase signals consistent with an increase in amyloidogenic processing of APP (Fig. 5a). To complement these assays, we also monitored $A \beta$ (1-38), $A \beta(1-40)$ and $A \beta(1-42)$ levels in 
(See figure on previous page.)

Fig. 4 Mutation of endogenous Drosophila KLC serine-433 to mimic permanent phosphorylation inhibits axonal transport of APP in wing sensory neurons. (a) Alignment showing high conservation of the amino acid sequences encompassing rat KLC1 serine-460 and the homologous Drosophila KLC serine-433 (indicated in red). (b) CRISPR genome editing approach to mutate KLC serine-433 to aspartic acid. Drosophila KIc sequence (upper line) along with the ssODN (lower line) are shown. Serine-433 codon (KLC) and aspartic acid codon (ssODN) are shown in red, the protospacer sequence in grey shade and the PAM site in blue shade. A G-to-A transition is introduced to mutate the PAM. (c) Representative kymographs showing axonal transport of APP-YFP in Klcwt and KICS433D homozygous backgrounds in 2-day old Drosophila; scale bar and times are indicated. Bar chart shows the relative proportions of stationary, anterograde and retrograde moving APP-YFP in the Klcwt background. (d) Bar charts show total, anterograde and retrograde APP-YFP movement runs in Klcwt and KICS433D backgrounds. (e) Violin plots show velocities of APP-YFP runs in anterograde and retrograde directions in the different backgrounds. Median and interquartile ranges are indicated by hashed lines. $N=8$ wings for each genotype. For velocity studies, $N=577$ anterogradely and 347 retrogradely moving APP-YFP in Klcwt, and 343 anterogradely and 247 retrogradely moving APP-YFP in the KICS433D background. Statistical significance was determined by one-way ANOVA with Holm-Sidak's multiple comparison test in (c), Mann-Whitney $U$ test in (d) and two-tailed Student's t test in (e). Error bars are s.e.m.; ${ }^{* *} \mathrm{p}<0.01$; ${ }^{* * *} p<0.0001$; ns not significant

conditioned media of HEK293 cells co-transfected with $\mathrm{APP}+\mathrm{KLC} 1 w \mathrm{wt}$ or APP+KLC1S460D. These assays revealed that expression of KLC1S460D significantly increased the levels of all these $A \beta$ species (Fig. 5b).

\section{Discussion}

Damage to axonal transport is an early pathogenic event in Alzheimer's disease. APP represents a key axonal transport cargo since disruption to its transport promotes amyloidogenic processing of APP and altered APP processing itself damages axonal transport [28, 49, 50, 56]. Understanding how APP is transported through axons and how this transport is regulated are therefore important aspects of Alzheimer's disease research.

APP is transported anterogradely through axons on kinesin-1 motors and a number of routes by which APP attaches to the motor have been described [7, 17, 24, 61]. One that has been linked directly to both APP transport and APP processing involves the KLC ligand calsyntenin-1 [11, $13,30,49,56]$. Here, APP is loaded onto calsyntenin-1 containing vesicles for transport by kinesin-1. Key regulatory events for this transport involve control of APP loading into the vesicle, and control of release of calsyntenin-1 (and hence vesicle associated APP) from KLCs.

Loading of APP onto calsyntenin-1 containing vesicles is believed to involve $\mathrm{X} 11 \beta$, an adaptor coat protein located in the Golgi that interacts with both APP and calsyntenin-1 [3-5, 12, 21, 26, 35, 48, 56]. Thus, manipulating expression of $\mathrm{X} 11 \beta$ influences both APP axonal transport and its amyloidogenic processing [25, 29, 47]. The mechanisms regulating release of APP from kinesin-1 motors are less well characterised. However, phosphorylation of KLC1 serine-460 has been shown to inhibit binding and axonal transport of calsyntenin-1 [55]. Here, via the use of phospho-mimicking mutants, we provide evidence that KLC1 serine-460 phosphorylation also inhibits axonal transport of APP.

The KLC1S460D mutant we utilise in rat cortical neurons has already been shown to accurately mimic the effects of KLC1 serine-460 phosphorylation on binding and axonal transport of calsyntenin-1 [55]. We show that expression of mutant KLC1S460D also inhibits axonal transport of APP in these rat neurons. We also demonstrate that mutation of its Drosophila homologue
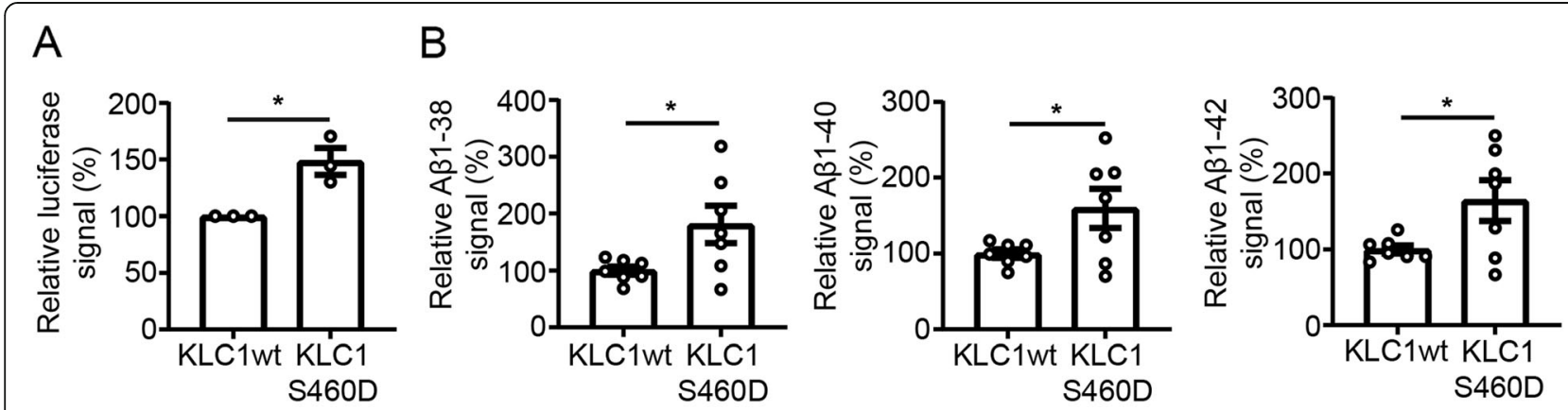

Fig. 5 Expression of KLC1 serine-460 to mimic permanent phosphorylation promotes amyloidogenic processing of APP. (a) APP processing was monitored in HEK293 cells essentially as described using an APP-GAL4 reporter assay that drives GAL4-UAS-dependent expression of firefly luciferase [22]. Luciferase signals were normalised to co-transfected Renilla signals to correct for transfection efficiency. Bar chart shows relative luciferase signals in cells transfected with the GAL4-UAS-luciferase+Renilla reporters plus either APP-GAL4 + KLC1wt or APP-GAL4 + KLC1S460D as indicated. $N=15$ from 3 independent experiments. Data were analysed by Student's t test, error bars are s.e.m., ${ }^{*} p<0.05$. (b) Expression of KLC1S460D increases $A \beta$ production. Levels of $A \beta$ (1-38), $A \beta(1-40)$ and $A \beta(1-42)$ we quantified in conditioned media from cells co-transfected with APP+KLC1wt or APP+ KLC1S460D. $N=7$; data were analysed by Student's t test, error bars are s.e.m., ${ }^{*} p<0.05$ 
KLC serine-433 to aspartate disrupts APP transport in vivo in Drosophila sensory neurons. In the cortical neurons, KLC1S460D reduced total (anterograde plus retrograde) transport but whilst both anterograde and retrograde transport appeared lower in the presence of KLC1S460D, these directional decreases did not reach significance. In Drosophila, KLCS433D reduced both total and anterograde axonal transport of APP. The more potent effect of KLCS433D on APP transport in Drosophila may be because the mutation is engineered into endogenous $K l c$ whereas in the cortical neurons, the approach involved exogenous KLC1S460D so permitting additional kinesin-1 transport via endogenous non-phosphorylated KLC1.

Whilst we detected reductions in the proportions of moving APP in both the KLC1 phospho-mimicking rat cortical and transgenic Drosophila neurons, we did not observe any changes to the velocities of APP transport in either system. Such observations are consistent with known role of KLC1 serine-460 phosphorylation in the attachment of cargo (calsyntenin-1) as opposed to any modulation of kinesin-1 motor function [55].

We also present evidence that phosphorylation of KLC1 serine-460 affects amyloidogenic processing of APP. Using an APP-GAL4-dependent firefly luciferase reporter assay, we show that expression of KLC1S460D promotes $\gamma$-secretase processing of APP. We also show that KLC1S460D expression increases production of $A \beta$ (1-38), $A \beta(1-40)$ and $A \beta(1-42)$ species. These findings are in line with the effects of KLC1S460D on APP transport since disruption to APP transport promotes amyloidogenic processing of APP $[49,50,56]$. Thus, phosphorylation of KLC1 serine-460 inhibits APP axonal transport and promote amyloidogenic APP processing.

Aside from ERK mediated phosphorylation of KLC1 serine-460, phosphorylation of other sites in KLCs have been shown to regulate axonal transport. Glycogen synthase kinase-3 $\beta$ (GSK3 $\beta$ ) phosphorylation of a C-terminal region of KLC2 has been linked to reductions in transport of vesicles and other cargoes [33, 38]. GSK3 $\beta$ may also phosphorylate KLC1 although no sites have yet been identified [37]. However, other studies suggest that any effects of GSK3 $\beta$ on APP transport involve altering the activity of kinesin-1 motors rather than any changes to KLCs [60]. Phosphorylation of KLC1 threonine-466 has also been shown to influence transport of APP [8]. The kinase that targets KLC1 threonine-466 is not known but once identified, it will be interesting to investigate whether there are linked signaling pathways that regulate KLC1 threonine466 and the nearby serine- 460 site phosphorylated by ERK that we investigate here.

Finally, we show that KLC1 levels are reduced and the relative levels of KLC1 serine-460 phosphorylation are increased in Alzheimer's disease frontal cortex. Reduced levels of both KLC1 and KLC2 have been described in late stage Alzheimer's disease cortex (Braak stage V/VI) [37]. Our results thus complement these findings and show that loss of KLC1 occurs in earlier Braak stage cases; early pathogenic events are believed to be the most relevant to disease. There is also evidence that phosphorylation of KLC1 is increased in Alzheimer's disease cortex although such studies have not identified altered phosphorylation of any specific residues [37]. Our findings are therefore the first to identify changes in phosphorylation of a defined site in kinesin-1 motor complexes in Alzheimer's disease brain (KLC1 serine460). Since we also present evidence that KLC1 serine460 phosphorylation affects axonal transport and processing of APP, our results suggest that increased KLC1 serine-460 phosphorylation contributes to the pathogenesis of Alzheimer's disease.

\section{Conclusions}

Damage to axonal transport of APP is believed to contribute to the pathogenesis of Alzheimer's disease. APP is transported anterogradely through axons on kinesin-1 motors and one route involves loading of APP into calsyntenin-1 containing vesicles; calsyntenin-1 is direct ligand for KLC1. The calsyntenin-1-KLC1 interaction is regulated by phosphorylation of KLC1 serine-460; phosphorylation promotes release of calsyntenin-1. Here we show that the expression of a phospho-mimicking mutant of KLC1S460 disrupts axonal transport of APP in rat cortical neurons and that a similar mutation disrupts transport of APP in vivo in Drosophila sensory wing neurons. We also demonstrate that the KLC1S460 mutant promotes amyloidogenic processing of APP. Finally, we show that KLC1 levels are reduced and the relative levels of KLC1 serine-460 phosphorylation are increased in Alzheimer's disease frontal cortex, and that this occurs relatively early in the disease process. Our results suggest that increased KLC1 serine-460 phosphorylation contributes to Alzheimer's disease.

\footnotetext{
Abbreviations

ANOVA: analysis of variance; APP: amyloid precursor protein; A $\beta$ : amyloid $\beta$ peptide; BACE1: $\beta$-site APP cleaving enzyme-1; CRISPR: type II clustered regularly interspaced short palindromic repeat; CRISPR-associated: Cas; EDTA: Ethylenediaminetetraacetic acid; EGFP: enhanced green fluorescent protein; ERK: extracellular-signal-regulated kinase; GAL4-UAS: GAL4 upstream activator sequence; gRNA: guide RNA; GSK3 3 : Glycogen synthase kinase-3ß; HEK293 cells: human embryonic kidney 293 cells; HRP: horseradish peroxidase; KLC: kinesin-1 light chains; KLC1S460A: kinesin light chain-1 serine-460 alanine mutant; KLC1S460D: kinesin light chain-1 serine-460 aspartic acid mutant; KLC1wt: wild-type kinesin light chain-1; KLCS433D: kinesin light chain serine-433 aspartic acid mutant; NSE: neuron specific enolase; OA: okadaic acid; PAM: protospacer adjacent motif; RIPA: radioimmunoprecipitation assay; s.e.m.: standard error of mean; SD: standard deviation; SDS-PAGE: sodium dodecyl sulphate polyacrylamide gel electrophoresis; ssODN: single stranded DNA oligonucleotide donor; TBS: Tris-HCl buffered saline; UAS: upstream activation sequence; YFP: yellow fluorescent protein
} 


\section{Acknowledgements}

We thank the Wohl Cellular Imaging Centre at King's College London for help with microscopy and the Fly Facility of the Department of Genetics, University of Cambridge for help with Drosophila embryo injections and Simon Bullock and Sean Munro (MRC-LMB Cambridge) for support to AV and NM.

\section{Authors' contributions}

CCJM, AV, EBG, GMM and WN designed the study. CCJM, AV, EBG and GMM wrote the manuscript. EBG, GMM and AV performed most experiments and analysed data; JG, DHWL, NB, FM and NM performed experiments and assisted in providing reagents. All authors edited the manuscript.

\section{Funding}

This work was supported by grants from Alzheimer's Research UK (ARTPG2011-5, ARUK-PG2014-5, ARUK-PG2017B-3 and ARUK-EG2013B-1 to CCJM and ARUK-RF2015-5 to EBG), a UK BBSRC grant (BB/L019299/1 to CCJM), a UK MRC grant (MR/R022666/1 to CCJM), NC3Rs David Sainsburys fellowship and SKT grants (N/N001753/2 and NC/T001224/1 to AV), an Academy of Medical Sciences Springboard Award (SBF004/1088 to AV) and a van Geest Fellowship in Dementia and Neurodegeneration and van Geest PhD studentship awards to AV.

\section{Availability of data and materials}

Experimental tools and data are available from the corresponding authors.

\section{Ethics approval and consent to participate}

Post-mortem human brain tissues were obtained from the MRC Neurodegenerative Diseases Brain Bank, King's College London. All tissue collection and processing were carried out under the regulations and licensing of the Human Tissue Authority, and in accordance with the Human Tissue Act, 2004

\section{Consent for publication}

Not applicable.

\section{Competing interests}

The authors declare they have no competing interests.

\section{Author details}

'Department of Basic and Clinical Neuroscience, Institute of Psychiatry, Psychology and Neuroscience, King's College London, 125 Coldharbour Lane Camberwell, London SE5 9RX, UK. ${ }^{2}$ Division of Cell Biology, MRC Laboratory of Molecular Biology, Cambridge CB2 OQH, UK.

\section{Received: 19 November 2019 Accepted: 25 November 2019}

Published online: 05 December 2019

\section{References}

1. Ackerley S, Thornhill P, Grierson AJ, Brownlees J, Anderton BH, Leigh PN Shaw CE, Miller CCJ (2003) Neurofilament heavy chain side-arm phosphorylation regulates axonal transport of neurofilaments. J Cell Biol 161:489-495

2. Ando K, Oishi M, Takeda S, lijima K, Isohara T, Nairn AC, Kirino Y, Greengard P, Suzuki T (1999) Role of phosphorylation of Alzheimer's amyloid precursor protein during neuronal differentiation. J Neurosci 19:4421-4427

3. Araki Y, Tomita S, Yamaguchi H, Miyagi N, Sumioka A, Kirino Y, Suzuki T (2003) Novel cadherin-related membrane proteins, Alcadeins, enhance the X11-like protein-mediated stabilization of amyloid beta -protein precursor metabolism. J Biol Chem 278:49448-49458

4. Araki Y, Kawano T, Taru H, Saito Y, Wada S, Miyamoto K, Kobayashi H, Ishikawa HO, Ohsugi Y, Yamamoto T et al (2007) The novel cargo Alcadein induces vesicle association of kinesin-1 motor components and activates axonal transport. EMBO J 26:1475-1486

5. Borg J-P, Ooi J, Levy E, Margolis B (1996) The phosphotyrosine interaction domains of $X 11$ and Fe65 bind to distinct sites on the YENPTY motif of amyloid precursor protein. Mol Cell Biol 16:6229-6241

6. Brady ST, Morfini GA (2017) Regulation of motor proteins, axonal transport deficits and adult-onset neurodegenerative diseases. Neurobiol Dis 105:273282. https://doi.org/10.1016/j.nbd.2017.04.010
7. Chiba K, Araseki M, Nozawa K, Furukori K, Araki Y, Matsushima T, Nakaya T, Hata S, Saito Y, Uchida S et al (2014) Quantitative analysis of APP axonal transport in neurons- role of JIP1 in enhanced APP anterograde transport. Mol Biol Cell. https://doi.org/10.1091/mbc. E14-06-1111

8. Chiba K, Chien KY, Sobu Y, Hata S, Kato S, Nakaya T, Okada Y, Nairn AC, Kinjo M, Taru $\mathrm{H}$ et al (2017) Phosphorylation of KLC1 modifies interaction with JIP1 and abolishes the enhanced fast velocity of APP transport by kinesin-1. Mol Biol Cell 28:3857-3869. https://doi.org/10. 1091/mbc. E17-05-0303

9. De Vos KJ, Chapman AL, Tennant ME, Manser C, Tudor EL, Lau KF, Brownlees J, Ackerley S, Shaw PJ, McLoughlin DM et al (2007) Familial amyotrophic lateral sclerosis-linked SOD1 mutants perturb fast axonal transport to reduce axonal mitochondria content. Hum Mol Genet 16: $2720-2728$

10. De Vos KJ, Grierson AJ, Ackerley S, Miller CCJ (2008) Role of axonal transport in neurodegenerative diseases. Annu Rev Neurosci 31:151-173

11. Dinamarca MC, Raveh A, Schneider A, Fritzius T, Fruh S, Rem PD, Stawarski M, Lalanne T, Turecek R, Choo M et al (2019) Complex formation of APP with GABAB receptors links axonal trafficking to amyloidogenic processing. Nat Commun 10:1331. https://doi.org/10.1038/s41467-019-09164-3

12. Dodding MP, Mitter R, Humphries AC, Way M (2011) A kinesin-1 binding motif in vaccinia virus that is widespread throughout the human genome. EMBO J 30:4523-4538. https://doi.org/10.1038/emboj.2011.326

13. Eggert S, Thomas C, Kins S, Hermey G (2018) Trafficking in Alzheimer's disease: modulation of APP transport and processing by the transmembrane proteins LRP1, SorLA, SorCS1C, Sortilin, and Calsyntenin. Mol Neurobiol 55:5809-5829. https://doi.org/10.1007/s12035-017-0806-x

14. Eidenmuller J, Fath T, Hellwig A, Reed J, Sontag E, Brandt R (2000) Structural and functional implications of tau hyperphosphorylation: information from phosphorylation-mimicking mutated tau proteins. Biochemistry 39:13166-13175

15. Encalada SE, Goldstein LS (2014) Biophysical challenges to axonal transport: motor-cargo deficiencies and neurodegeneration. Annu Rev Biophys 43 141-169. https://doi.org/10.1146/annurev-biophys-051013-022746

16. Falzone TL, Gunawardena S, McCleary D, Reis G, Goldstein LS (2010) Kinesin1 transport reductions enhance human tau hyperphosphorylation, aggregation and neurodegeneration in animal models of tauopathies. Hum Mol genet 19:4399-4408

17. Fu MM, Holzbaur EL (2013) JIP1 regulates the directionality of APP axonal transport by coordinating kinesin and dynein motors. J Cell Biol. https://doi. org/10.1083/jcb.201302078

18. Gauger AK, Goldstein LS (1993) The Drosophila kinesin light chain. Primary structure and interaction with kinesin heavy chain. J Biol Chem 268:1365713666

19. Gibbs KL, Greensmith L, Schiavo G (2015) Regulation of axonal transport by protein kinases. Trends Biochem Sci 40:597-610. https://doi.org/10.1016/j. tibs.2015.08.003

20. Gunawardena S, Her LS, Brusch RG, Laymon RA, Niesman IR, Gordesky-Gold B, Sintasath L, Bonini NM, Goldstein LS (2003) Disruption of axonal transport by loss of huntingtin or expression of pathogenic polyQ proteins in Drosophila. Neuron 40:25-40

21. Hill K, Li Y, Bennett M, McKay M, Zhu X, Shern J, Torre E, Lah JJ, Levey Al, Kahn RA (2003) Munc18 interacting proteins: ADP-ribosylation factordependent coat proteins that regulate the traffic of beta-Alzheimer's precursor protein. J Biol Chem 278:36032-36040

22. Hoey SE, Williams RJ, Perkinton MS (2009) Synaptic NMDA receptor activation stimulates alpha-secretase amyloid precursor protein processing and inhibits amyloid-beta production. J Neurosci 29:4442-4460

23. Kaether C, Skehel P, Dotti CG (2000) Axonal membrane proteins are transported in distinct carriers: a two-color video microscopy study in cultured hippocampal neurons. Mol Biol Cell 11:1213-1224

24. Kamal A, Stokin GB, Yang ZH, Xia CH, Goldstein LSB (2000) Axonal transport of amyloid precursor protein is mediated by direct binding to the kinesin light chain subunit of kinesin-I. Neuron 28:449-459

25. Kondo M, Shiono M, Itoh G, Takei N, Matsushima T, Maeda M, Taru H, Hata S, Yamamoto T, Saito $Y$ et al (2010) Increased amyloidogenic processing of transgenic human APP in X11-like deficient mouse brain. Mol Neurodegener 5:35

26. Konecna A, Frischknecht R, Kinter J, Ludwig A, Steuble M, Meskenaite V, Indermuhle M, Engel M, Cen C, Mateos JM et al (2006) Calsyntenin-1 docks vesicular cargo to kinesin-1. Mol Biol Cell 17:3651-3663 
27. Kurbatskaya K, Phillips EC, Croft CL, Dentoni G, Hughes MM, Wade MA, AlSarraj S, Troakes C, O'Neill MJ, Perez-Nievas BG et al (2016) Upregulation of calpain activity precedes tau phosphorylation and loss of synaptic proteins in Alzheimer's disease brain. Acta Neuropathol Commun 4:34. https://doi. org/10.1186/s40478-016-0299-2

28. Lazarov O, Morfini GA, Pigino G, Gadadhar A, Chen X, Robinson J, Ho H, Brady ST, Sisodia SS (2007) Impairments in fast axonal transport and moto neuron deficits in transgenic mice expressing familial Alzheimer's diseaselinked mutant presenilin 1. J Neurosci 27:7011-7020

29. Lee JH, Lau KF, Perkinton MS, Standen CL, Rogelj B, Falinska A, McLoughlin DM, Miller CC (2004) The neuronal adaptor protein X11 beta reduces Abeta levels and amyloid plaque formation in the brains of transgenic mice. J Biol Chem 279:49099-49104

30. Ludwig A, Blume J, Diep TM, Yuan J, Mateos JM, Leuthauser K, Steuble M, Streit P, Sonderegger P (2009) Calsyntenins mediate TGN exit of APP in a kinesin-1-dependent manner. Traffic (Copenhagen, Denmark) 10:572-589

31. Maeder Cl, Shen K, Hoogenraad CC (2014) Axon and dendritic trafficking. Curr Opin Neurobiol 27c:165-170. https://doi.org/10.1016/j.conb.2014.03.015

32. Mangeol P, Prevo B, Peterman EJ (2016) KymographClear and KymographDirect: two tools for the automated quantitative analysis of molecular and cellular dynamics using kymographs. Mol Biol Cell 27: 1948-1957

33. Manser C, Guillot F, Vagnoni A, Davies J, Lau KF, McLoughlin DM, De Vos KJ Miller CC (2012) Lemur tyrosine kinase-2 signalling regulates kinesin-1 light chain-2 phosphorylation and binding of Smad2 cargo. Oncogene 31:27732782. https://doi.org/10.1038/onc.2011.437

34. Manser C, Vagnoni A, Guillot F, Davies J, Miller CCJ (2012) Cdk5/p35 phosphorylates lemur tyrosine kinase-2 to regulate protein phosphatase-1C phosphorylation and activity. J Neurochem 121:343-348. https://doi.org/10. 1111/j.1471-4159.2012.07650.x

35. McLoughlin DM, Miller CCJ (1996) The intracellular cytoplasmic domain of the Alzheimer's disease amyloid precursor protein interacts with phosphotyrosine binding domain proteins in the yeast two-hybrid system. FEBS Lett 397:197-200

36. Millecamps S, Julien JP (2013) Axonal transport deficits and neurodegenerative diseases. Nat Rev Neurosci 14:161-176. https://doi.org/ 10.1038/nrn3380

37. Morel M, Heraud C, Nicaise C, Suain V, Brion JP (2012) Levels of kinesin light chain and dynein intermediate chain are reduced in the frontal cortex in Alzheimer's disease: implications for axoplasmic transport. Acta Neuropathol 123:71-84. https://doi.org/10.1007/s00401-011-0901-4

38. Morfini G, Szebenyi G, Elluru R, Ratner N, Brady ST (2002) Glycogen synthase kinase 3 phosphorylates kinesin light chains and negatively regulates kinesin-based motility. EMBO J 21:281-293

39. Morfini G, Schmidt N, Weissmann C, Pigino G, Kins S (2016) Conventional kinesin: biochemical heterogeneity and functional implications in health and disease. Brain Res Bull 126:347-353. https://doi.org/10.1016/j. brainresbull.2016.06.009

40. Morotz GM, De Vos KJ, Vagnoni A, Ackerley S, Shaw CE, Miller CCJ (2012) Amyotrophic lateral sclerosis-associated mutant VAPBP56S perturbs calcium homeostasis to disrupt axonal transport of mitochondria. Hum Mol Genet 21:1979-1988. https://doi.org/10.1093/hmg/dds011

41. Morotz GM, Glennon EB, Gomez-Suaga P, Lau DHW, Robinson ED, Sedlak E, Vagnoni A, Noble W, Miller CCJ (2019) LMTK2 binds to kinesin light chains to mediate anterograde axonal transport of cdk5/p35 and LMTK2 levels are reduced in Alzheimer's disease brains. Acta Neuropathol Commun 7:73. https://doi.org/10.1186/s40478-019-0715-5

42. Neumann S, Chassefeyre R, Campbell GE, Encalada SE (2016) KymoAnalyzer: a software tool for the quantitative analysis of intracellular transport in neurons. Traffic, Copenhagen. https://doi.org/10.1111/tra.12456

43. Perkinton MS, Standen CL, Lau KF, Kesavapany S, Byers HL, Ward M, McLoughlin DM, Miller CCJ (2004) The c-Abl tyrosine kinase phosphorylates the Fe65 adaptor protein to stimulate Fe65/amyloid precursor protein nuclear signalling. J Biol Chem 279:22084-22091

44. Port F, Chen HM, Lee T, Bullock SL (2014) Optimized CRISPR/Cas tools for efficient germline and somatic genome engineering in Drosophila. Proc Natl Acad Sci U S A 111:E2967-E2976

45. Reis GF, Yang G, Szpankowski L, Weaver C, Shah SB, Robinson JT, Hays TS, Danuser G, Goldstein LS (2012) Molecular motor function in axonal transport in vivo probed by genetic and computational analysis in Drosophila. Mol Biol Cell 23:1700-1714
46. Richardson CD, Ray GJ, DeWitt MA, Curie GL, Corn JE (2016) Enhancing homology-directed genome editing by catalytically active and inactive CRISPR-Cas9 using asymmetric donor DNA. Nat Biotechnol 34:339-344. https://doi.org/10.1038/nbt.3481

47. Saito Y, Akiyama M, Araki Y, Sumioka A, Shiono M, Taru H, Nakaya T, Yamamoto T, Suzuki T (2011) Intracellular trafficking of the amyloid betaprotein precursor (APP) regulated by novel function of X11-like. PLoS One 6: e22108. https://doi.org/10.1371/journal.pone.0022108

48. Shrivastava-Ranjan P, Faundez V, Fang G, Rees H, Lah JJ, Levey Al, Kahn RA (2008) Mint3/X11gamma is an Arf-dependent adaptor that regulates the traffic of the Alzheimer's precursor protein from the TGN. Mol Biol Cell 19:51-64

49. Steuble M, Diep TM, Schatzle P, Ludwig A, Tagaya M, Kunz B, Sonderegger P (2012) Calsyntenin-1 shelters APP from proteolytic processing during anterograde axonal transport. Biol Open 1:761-774. https://doi.org/10.1242/ bio.20121578

50. Stokin GB, Lillo C, Falzone TL, Brusch RG, Rockenstein E, Mount SL, Raman R, Davies P, Masliah E, Williams DS et al (2005) Axonopathy and transport deficits early in the pathogenesis of Alzheimer's disease. Science (New York, NY) 307:1282-1288

51. Szodorai A, Kuan YH, Hunzelmann S, Engel U, Sakane A, Sasaki T, Takai Y, Kirsch J, Muller U, Beyreuther K et al (2009) APP anterograde transport tequires Rab3A GTPase activity for assembly of the transport vesicle. J Neurosci 29:14534-14544

52. Tiwari SS, Mizuno K, Ghosh A, Aziz W, Troakes C, Daoud J, Golash V, Noble W, Hortobagyi T, Giese KP (2016) Alzheimer-related decrease in CYFIP2 links amyloid production to tau hyperphosphorylation and memory loss. Brain 139:2751-2765. https://doi.org/10.1093/brain/aww205

53. Vagnoni A, Bullock SL (2016) A simple method for imaging axonal transport in aging neurons using the adult Drosophila wing. Nat Protoc 11:17111723. https://doi.org/10.1038/nprot.2016.112

54. Vagnoni A, Bullock SL (2018) A CAMP/PKA/Kinesin-1 axis promotes the axonal transport of mitochondria in aging Drosophila neurons. Curr biol 28: 1265-1272.e1264. https://doi.org/10.1016/j.cub.2018.02.048

55. Vagnoni A, Rodriguez L, Manser C, De Vos KJ, Miller CCJ (2011) Phosphorylation of kinesin light chain-1 at serine-460 modulates binding and trafficking of calsyntenin-1. J Cell Sci 124:1032-1042

56. Vagnoni A, Perkinton MS, Gray EH, Francis PT, Noble W, Miller CCJ (2012) Calsyntenin-1 mediates axonal transport of the amyloid precursor protein and regulates Abeta production. Hum Mol Genet 21:2845-2854. https://doi. org/10.1093/hmg/dds109

57. Vagnoni A, Glennon EB, Perkinton MS, Gray EH, Noble W, Miller CC (2013) Loss of c-Jun N-terminal kinase-interacting protein-1 does not affect axonal transport of the amyloid precursor protein or Abeta production. Hum Mol Genet 22:4646-4652. https://doi.org/10.1093/hmg/ddt313

58. Vagnoni A, Hoffmann PC, Bullock SL (2016) Reducing Lissencephaly-1 levels augments mitochondrial transport and has a protective effect in adult Drosophila neurons. J Cell Sci 129:178-190

59. van der Kant R, Goldstein LS (2015) Cellular functions of the amyloid precursor protein from development to dementia. Dev Cell 32:502-515. https://doi.org/10.1016/j.devcel.2015.01.022

60. Weaver C, Leidel C, Szpankowski L, Farley NM, Shubeita GT, Goldstein LS (2013) Endogenous GSK-3/shaggy regulates bidirectional axonal transport of the amyloid precursor protein. Traffic (Copenhagen, Denmark) 14:295-308. https://doi.org/10.1111/tra.12037

61. Zheng P, Eastman J, Vande Pol S, Pimplikar SW (1998) PAT1, a microtubuleinteracting protein, recognizes the basolateral sorting signal of amyloid precursor protein. Proc Natl Acad Sci U S A 95:14745-12750

\section{Publisher's Note}

Springer Nature remains neutral with regard to jurisdictional claims in published maps and institutional affiliations. 